\title{
Publisher Correction: The impact of endothermy on the climatic niche evolution and the distribution of vertebrate diversity
}

\author{
Jonathan Rolland (D), Daniele Silvestro (D), Dolph Schluter, Antoine Guisan, Olivier Broenniman and Nicolas Salamin
}

Correction to: Nature Ecology \& Evolution https://doi.org/10.1038/s41559-017-0451-9, published online 29 January 2018.

In the version of this Article originally published, in Fig. 3a the first boundary was incorrectly labelled the "K/T boundary"; it should have read the "K/Pg boundary". The two equations in the main text were incorrectly omitted from the HTML. In the description of the posterior distribution of an ancestral state, the normal distribution was incorrectly described as being "assigned as prior to the node value"; it should have read "assigned as calibration to the node value". In the associated equation (the second equation in the text), the denominator of the last term was incorrectly given as "Node prior"; it should have read "Node calibration". In the same equation, the numerator of the third term on the right-hand side of the equation contained incorrect superscript notation on the $x$ and should have been: $\mathcal{N}\left(x_{i+1}^{\prime \prime}, \sigma^{2} t_{3}\right)$, as shown in the full equation below.

$$
\underbrace{x_{i}}_{\text {Posterior }} \sim \underbrace{\mathcal{N}\left(x_{i-1}, \sigma^{2} t_{1}\right)}_{\text {Ancestor }} \times \underbrace{\mathcal{N}\left(x_{i+1}^{\prime}, \sigma^{2} t_{2}\right)}_{\text {Descendant 1 }} \times \underbrace{\mathcal{N}\left(x_{i+1}^{\prime \prime}, \sigma^{2} t_{3}\right)}_{\text {Descendant 2 }} \times \underbrace{\mathcal{N}\left(\mu_{i}, \sigma_{i}^{2}\right)}_{\text {Node calibration }}
$$

In the Acknowledgements, the following two sentences were incorrectly omitted: “The authors thank the Vital-IT facilities of the Swiss Institute of Bioinformatics for the computational support" and "This work was funded by the University of Lausanne and the Swiss National Science Foundation (CRSIII3-147630) to N.S." In the Author contributions section, the first sentence was incorrectly given as "J.R. designed the study. J.R., N.S. and D. Silvestro designed the methodology and ran the analyses"; it should have read "J.R., D.S. and N.S. designed the study and the methodology". In the Supplementary Information, all three instances of the word "prior" were incorrect and should have read "calibration".

These errors have now been corrected in all versions of the Article. 